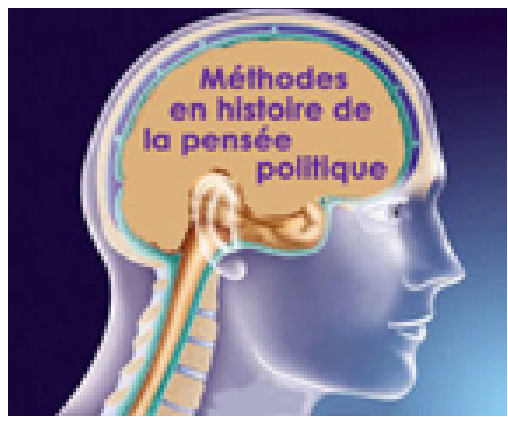

\section{3-24 septembre 2004}

Colloque de l'Association Française de Science politique, sousla direction de Lucien J aume, directeur de recherche au CNRS (CEVIPOF)

Avec le soutien du Ministère délégué à la Recherche, de la Fondation nationale des Sciences Politiques (FNPSP), du Centre de Recherches Politiques de Sciences Po (CEVIPOF) et du Centre d'Histoire du Ving tième Siècle (CHEVS)

\title{
Méthodes en histoire de la pensée politique
}

\section{Lieu : FNSP - 56 rue J acob 75006 Paris} France (salle de conférences)

\author{
En savoir plus sur le \\ ww w.afsp.msh-paris.fr
}

Une rencontre sur l'histoire de la pensée politique considérée sous l'angle épistémologique ou méthodologique

Ce colloque prend acte de la richesse actuelle du débat intemational via la recherche de nouvelles: méthodes et de nouveaux objets dans des domai- : nes aussi différents que l'histoire des concepts, l'his : toire intellectuelle, l'histoire de la philosophie politique, ou encore la théorie juridique dans ses rap- : ports avec l'histoire du droit. Les paradigmes qui : éta ient importants il y a une trenta ine d'a nnées sont maintenant discutés sur leur propre terrain et la is sent apparaître des formulations concurrentes. La question de la dimension historique de la philosophie (philosophie en général ou philosophie poli- : tique), de sa spécific ité ou de son intégration à l'histoire desidées, est de nouveau remise en chantier.
Une réflexion sur: les pratiques recherches

Souhaitant faire appa raître des problèmes de méthode, expérimentés et réfléchis par: ceux-là même qui tentent de saisir la pensée politique dans une : dimension historique, la réflexion insistera sur les: pratiques de recherche telles qu'elles existent, à la fois dans leur diversité et à l'intérieur de disciplines différentes.

\section{jeudi 23 septembre 2004}

Introduction par Lucien J aume : Les perspectives générales

Axe $n^{\circ} 1$ : A quel objet l'historien de la pensée politique a-t-il affaire ? - La métaphore dans la représentation du pouvoir - Pietro COSTA, professeur d'histoire du droit à l'Université de Florence.

I Qu'est-ce qu'écrire un dictionnaire des concepts politiques en historien ? - Javier Femández SEBASTIÁN, prof. d'Histoire de la pensée et des mouvements socia ux et politiques, Université de Bilbao.

I Pierre de Coubertin et l'invention de la neutralité politique du sport Patrick CLASTRES, maître de conférences en histoire, IEP et CHEVS.

Axe $n^{\circ} 2$ : Les régimes de vérité du discours historien

Président : Marc SADOUN (professeur, IEP de Paris, directeur du DEA Pensée Politique ")

I La loi et le parlementarisme : peut-on fédérer les approches historique, juridique et philosophique ? - Nicolas ROUSSEШER, maître de conférence, IEP de Paris

I Les frères De la Court et la tradition du républica nisme - Martin Van GELDEREN, professeur, Institut Universita ire Européen de Florence.

I L'histoire de la philosophie doit-elle être philosophique ? Charles COUTEL, professeur, doyen de la Faculté de droit et de science politique Alexis de Tocqueville, Doua i.

I La fiction littéraire comme accès au politique : voies d'interprétation. Françoise MÉLONIO, professeur, Université de Paris IV, directrice de l'UFR de lettres.

\section{Atelier Benjamin Constant}

Animation de l'atelier: Paul DEBOUUE, prof. honora ire, Université de Liège, président du Comité directeurpour l'éd ition des F uvrescomplètes de Benjamin Constant

I Le sens d'une entreprise : éditer tout Constant - Paul DELBOUIШE.

I Les problèmes du texte polyphonique : éditer, interpréter, commenter Etienne HOFMANN, professeur à I'Université de Lausanne, Président de I'Institut Benjamin Constant, membre du Comité directeur des OCBC

I Comment embrasser la diversité d'un auteur/acteur/fondateur? - Kurt KLOOCKE, professeur à I'Université de Tübingen, membre du Comité directeur des OCBC. une comparaison et un dialogue entre
des approches disciplinaires différentes
En questionna nt l'existence d'un "champ de
l'histoire de la pensée politique ", et sanspar-
tir d'une interdisciplina rité supposée comme
existante, il s'agira de construire un échan-
ge entre disciplines sans se dissimuler les
divergences. Mais l'un des gains de la com-
paraison est de progresser sur le site et la
nature même de ce que l'on appelle " pen-
sée politique "et de vénifier, à l'occasion des
recherches les plus récentes, qu'elle ne peut
plus être limitée au domaine des " grands
auteurs " ni à l'enchaînement supposé entre
les " idées ". plusieurs types de matériaux et
de questionnement permettent désomais
de saisir autrement la pensée politique.
....................................................... vendredi 24 septembre 2004

Axe $n^{\circ} 3$ : Les idées, avec les agents ? ou sans les agents?

Président : Serge BERSTEIN (professeur, IEP de Paris) I Mentalités, culture politique, idéologie - Marcel GAUCHET, directeur d'études à l'EHESS.

- Quel traitement de la pensée politique peut donner la sociologie historique du politique ? Yves DÉLOYE, professeur, Institut d'Etudes Politiques de Stra sbourg et Olivier IHL, professeur, Institut d'Etudes Politiques de Grenoble.

I Rhétorique de l'action et pensée politique - Kari PALONEN, professeur à l'Université de Jyväskylä (sujet proviso ire).

I Pourquoi Foucault, qui ne parle pas en philosophe, parle-t-il aujourd'hui au philosophe? Yves Charles ZARKA, directeur de recherche au CNRS, directeur du Centre de la philosophie modeme (CNRS, Villejuif).

Axe $n^{\circ} 4$ : Le concept et la dimension temporelle I Fin du concept de souveraineté ? - Jean-Pierre MACHELON, professeur, Université René Descartes Paris V, directeur d'études Ecole pratique des Hautes Etudes.

- Histoire constitutionnelle : quelques réflexions de méthode - Joaquin Varela SUANZES, professeur, Université d'Oviedo, directeur de Historia Constitucional.

I Le concept de république : continuités mythiques et continuités réelles - Janet COLEMAN, professeur, London School of Economics and Political Science.

Historic ité des concepts juridiques ? - Michel TROPER, professeur, Université Paris X Na ntemre

Conclusion générale

Rapporteurs: François DOSSE, Lucien J AUME 